Pacific Journal of Mathematics

A UNIFORMLY CONTINUOUS FUNCTION ON [0, 1$]$ THAT IS
EVERYWHERE DIFFERENT FROM ITS INFIMUM 


\title{
A UNIFORMLY CONTINUOUS FUNCTION ON $[0,1]$ THAT IS EVERYWHERE DIFFERENT FROM ITS INFIMUM
}

\author{
William JULian AND Fred RichmaN
}

\begin{abstract}
An example of a uniformly continuous function on $[0,1]$ that is everywhere different from its infimum is constructed in the context of Bishop's constructive mathematics using a consequence of Chruch's thesis. The existence of such a function is shown to be equivalent to the constructive denial of König's lemma. Conversely König's lemma is shown to be equivalent to the intuitionistic theorem that every positive uniformly continuous function on $[0,1]$ has a positive infimum. Various applications to constructive mathematics are given.
\end{abstract}

0. Introduction. Let $f$ be a uniformly continuous function on the closed unit interval $[0,1]$. Although the infimum of $f$ can be explicitly constructed, Brouwerian counterexamples preclude a general procedure for constructing a point at which this infimum is achieved. These counterexamples do not involve constructing a uniformly continuous function that is everywhere different from its infimum; in fact it is a theorem in intuitionistic mathematics, but not in Bishop's constructive mathematics, that an everywhere positive function has a positive infimum [5; Theorem 5, p. 69].

If one operates in the context of recursive function theory, and demands only pointwise continuity, then a famous construction of Specker [12] easily yields a continuous function $f$ which is different from its infimum at every (recursive) point in $[0,1]$. The question whether an everywhere positive uniformly continuous function on $[0,1]$ has a positive infimum was raised by Bishop [3; p. 151], and, in the context of recursive analysis, by Grzegorczyk [4].

A construction, from the point of view of recursive analysis, of a (recursively) uniformly continuous function on $[0,1]$ that is different from its infimum at every recursive point, was outlined by Kreisel [8] in a review of another paper. Aberth [1; Theorem 7.12] constructed such a function from the point of view of computable analysis: roughly Russian constructivism without quite the philosophical commitment-it may be identified, in a pinch, with recursive analysis. Zaslavsky [14; Theorem 5.5] constructed such a function in the context of Russian constructivism and 
Specker [13] gave a construction from the point of view of recursive function theory. Beeson gives a construction in his forthcoming book [2] on the metamathematics of constructive mathematics. Kreisel uses a predicate of Kleene which can be found in [7; Lemma 9.8] modulo a reference to [6], which gives an unbounded branch-bounded fan that he uses to construct his function. Aberth's construction relies on Markov's principle in the form that two real numbers are distinct (apart) if they cannot be equal. Lacombe [9] announced the existence of such a function but gave no construction.

In this paper we give a complete, self-contained, construction of a positive uniformly continuous function on $[0,1]$ with infimum zero (Corollary 2.5), without invoking Markov's principle, in the context of Bishop's constructive mathematics, assuming a consequence of Church's thesis. To the extent that Church's thesis does not admit a constructive refutation, this example precludes a constructive proof that a positive uniformly continuous function on $[0,1]$ has a positive infimum. Other similarly limiting examples, based on this one, are given in $\S 3$. We also show (Theorem 2.4), without assuming anything, that the existence of such a function is equivalent to the existence of an unbounded branch-bounded fan, that is, a counterexample to Brouwer's fan theorem. Conversely we show that the intuitionistically valid assertion that every branch-bounded fan is bounded is equivalent to the assertion that every positive, uniformly continuous function on $[0,1]$ has a positive infimum (Corollary 2.6).

The consequence of Church's thesis that we shall employ is the following:

CPF The set of partial functions is countable.

For a detailed exposition of CPF see [11]; we shall briefly indicate what it means. Let $N$ denote the set of positive integers. By a partial function algorithm we mean a function $A$ from $N \times N$ to $N \cup\{\perp\}$ such that if $A(x, n) \neq \perp$, then $A(x, n+1)=A(x, n)$. The partial function $f$ associated with $A$ is defined on $\operatorname{dom} f=\{x \in N ; A(x, n) \neq \perp$ for some $n\}$ by setting $f(x)=A(x, n)$ for any $n$ such that $A(x, n) \neq \perp$. One should think of $A(x, n)$ as being the result of running the algorithm $A$ for $n$ steps on the input $x$, with $\perp$ standing for no output. The axiom CPF says that there is a sequence $A_{1}, A_{2}, A_{3}, \ldots$ of partial function algorithms, with 
associated partial functions $f_{1}, f_{2}, f_{3}, \ldots$ such that given any partial function $g$ you can find a positive integer $m$ with $g=f_{m}$. If a computable function is one you can write a computer program for (Church's thesis), and if all functions are computable (Bishop's thesis), then CPF holds.

1. Binary fans. The complete binary fan $C$ is the set of all finite sequences $a=\left(a_{1}, a_{2}, \ldots, a_{n}\right)$ where $a_{i}= \pm 1$ and $n \in N$. We say that $a$ is a node, that $n$ is the length of $a$, and we write $n=|a|$. If $|a| \geq m$, then the restriction of $a$ to $m$ is the sequence $a_{1}, a_{2}, \ldots, a_{m}$ which is denoted by $a(m)$. A branch $a$ of $C$ is an infinite sequence $a_{1}, a_{2}, \ldots$; the restriction of a branch is defined in the same way as the restriction of a node.

A binary fan $F$ is a subset of $C$ that is closed under restriction. A fan $F$ is branch-bounded if each branch of $C$ has a restriction that is not in $F$. A fan $F$ is bounded if there is an integer $m \in N$ such that no element of $F$ has length exceeding $m$. From a classical point of view, every branch-bounded fan is bounded (König's lemma). From an intuitionistic point of view this follows from Brouwer's celebrated fan theorem [5; 3.4.4]. From a recursive function theoretic point of view this is false [7; Lemma 9.8]. We shall construct, in the context of Bishop's constructive mathematics with CPF, an unbounded, branch-bounded fan.

A subset $B$ of the complete binary fan $C$ is a bar if every branch of $C$ has a restriction in $B$. This notion is central to the intuitionistic development of Brouwer's fan theorem and continuity principle [5; 3.4]. In the second edition of [5] bars were not required to be detachable, while in the third edition they are; we shall adhere to the former usage. To every subset $B$ of $C$ there corresponds a fan $F$ consisting of those nodes of $C$ which have no restrictions in $B$. If $B$ is a bar, then $F$ is branch-bounded. We wish to construct a detachable branch-bounded fan $F$ containing nodes of arbitrary length. To do this we construct a certain detachable bar. First we construct a bar that need not be detachable, but whose associated fan cannot be bounded.

THEOREM 1.1. Assuming CPF there is a countable bar B that contains at most one node of each length.

Proof. Let $B=\left\{a \in C: a=\left(f_{m}(1), f_{m}(2), \ldots, f_{m}(m)\right)\right.$ for some $m \in N$ with $\left.1,2, \ldots, m \in \operatorname{dom}\left(f_{m}\right)\right\}$. Clearly $B$ is a bar as every branch is some (total) function $f_{m}$. To see that the bar $B$ is countable let $b_{0}$ be any element of $B$ and define $b_{m k}=\left(A_{m}(1, k), A_{m}(2, k), \ldots, A_{m}(m, k)\right)$ if each $A_{m}(i, k)$ is \pm 1 , and $b_{m k}=b_{0}$ otherwise. 
We now show how to construct a detachable bar with the desired property. More generally we have:

LEMMA 1.2. Let $B$ be a countable subset of $C$. Then there is a detachable subset $B^{\prime}$ of $C$ such that each element of $B^{\prime}$ has a restriction in $B$, and for each node $b$ of $B$ there is $n>|b|$ such that every node of length $n$ extending $b$ has a restriction in $B^{\prime}$. In particular, if $B$ is a bar, then so is $B^{\prime}$.

Proof. Let $c_{1}, c_{2}, \ldots$ be a one-to-one enumeration of $C$ and let $b_{1}, b_{2}, \ldots$ be an enumeration of $B$. Then

$$
B^{\prime}=\left\{c_{l}: c_{i}(m)=b_{J} \text { for some } j, m \in N \text { with } j \leq i\right\}
$$

is detachable and every element of $B^{\prime}$ has a restriction in $B$. If $a$ is a branch in $C$, then $a(m)=b_{j} \in B$ for some $m$ and $j$. If $n$ is sufficiently large, and $a(n)=c_{i}$, then $i \geq j$ so $c_{i} \in B^{\prime}$.

THEOREM 1.3. Assuming CPF there is a detachable branch-bounded fan that contains nodes of arbitrary length.

Proof. Let $B$ be the bar of Lemma 1.1 and $B^{\prime}$ the corresponding detachable bar of Lemma 1.2. The desired fan is $F=\left\{a \in C: a(m) \notin B^{\prime}\right.$ for all $m \leq|a|\}$. To show that $F$ contains a node of length $n$, suppose each node of length $n$ had a restriction in $B^{\prime}$. Then each node of length $n$ would have a restriction in $B$. But this is impossible as $B$ contains at most one node of each length not exceeding $n$.

The existence of a detachable branch-bounded fan containing nodes of arbitrary length is the essential content of [7; Lemma 9.8] which relies on a couple of predicates from [6; p. 308]. As that part of recursive function theory necessary for the proof of [7; Lemma 9.8] seems to be free of any invocation of Markov's principle, and Church's thesis only enters in the form of $\mathrm{CPF}$, this provides an alternative proof of Theorem 1.3.

2. Geometric realizations of fans. With each node $a$ of length $n$ of the complete binary fan $C$ we associate a point $\left(x_{a}, y_{a}\right)$ in the plane by setting

$$
\begin{aligned}
& x_{a}=\frac{1}{2}+\sum_{i=1}^{n} a_{i} 3^{-\imath} \\
& y_{a}=\frac{1}{2}-\sum_{i=1}^{n} 3^{-i}=\frac{3^{-n}}{2},
\end{aligned}
$$


and if $F$ is a subset of $C$ we set $P(F)=\left\{\left(x_{a}, y_{a}\right): a \in F\right\}$. Note that every point of $P(C)$ is isolated, so any finite subset of $P(C)$ is detachable. We set $x_{a(0)}=y_{a(0)}=1 / 2$.

THEOREM 2.1. A binary fan $F$ is detachable (from $C$ ) if and only if $P(F)$ is totally bounded.

Proof. If $F$ is detachable, then $F_{n}=\{a \in F:|a| \leq n\}$ is finite and $P\left(F_{n}\right)$ is a $3^{-n}$-approximation to $P(F)$. Conversely suppose $P(F)$ is totally bounded. Given a node $a$ in $C$ construct a finite $3^{-|a|-1}$-approximation $K$ to $P(F)$. As the distance from $P(a)$ to $P(C \backslash a)$ is $3^{-|a|-1} \sqrt{2}$, we have $a \in F$ if and only if $P(a) \in K$.

LEMMA 2.2. Let $x$ be a real number and $b$ be a branch of $C$. Suppose for some $n>0$ we have $b_{n}=1$ and $x_{b(n-1)}>x$, or $b_{n}=-1$ and $x_{b(n-1)}<x$. Then $\left|x_{b(m)}-x\right|>3^{-n} / 2$ for all $m \geq n$.

LEMMA 2.3. Let $x$ be a real number. Then there exists a branch a of $C$ such that for each binary fan $F$, if $a(m) \notin F$, then $(x, 0)$ is bounded away from $P(F)$ by $3^{-m} / 4$.

Proof. Construct $a$ such that $x_{a(n-1)}<x$ whenever $a_{n}=1$ and $x_{a(n-1)}$ $>x-3^{-n} / 5$ whenever $a_{n}=-1$. Suppose $F$ is a fan and $a(m) \notin F$. If $\left|x-x_{a(n-1)}\right|<3^{-n} / 4$ for some $n \leq m$, then $(x, 0)$ is bounded away from $P(C)$ by $3^{-n} / 4$. Otherwise for each $n \leq m$ we have $\left|x-x_{a(n-1)}\right|>3^{-n} / 5$, so $x_{a(n-1)}<x$ if $a_{n}=1$, and $x_{a(n-1)}>x$ if $a_{n}=-1$. As $a(m) \notin F$, if $b$ is a node of $F$ with $|b| \geq m$, then $b_{n} \neq a_{n}$ for some $n \leq m$. Thus $\left|x_{b}-x\right|>$ $3^{-n} / 2>3^{-m} / 4$ by Lemma 2.2. On the other hand, if $|b|<m$, then $\left|y_{b}\right|>3^{-m} / 2$.

THEOREM 2.4. Given a nonnegative, uniformly continuous function $f$ on $[0,1]$ we can construct a detachable fan $F$ such that

(1) $0<f(x)$ for all $x$ if and only if $F$ is branch-bounded,

(2) $0<\inf f$ if and only if $F$ is bounded.

Conversely, given a detachable fan $F$ we can construct a nonnegative, uniformly continuous function $f$ on $[0,1]$ satisfying (1) and (2).

Proof. Let $F$ be a detachable fan. Then $P(F)$ is totally bounded (Theorem 2.1) so we can compute the distance $f(x)$ from $(x, 0)$ to $P(F)$. Clearly $f$ is a nonnegative, uniformly continuous function. Let $x$ be a real 
number and $a$ the branch constructed in Lemma 2.3. If $F$ is branch-bounded, we can find $m$ such that $a(m) \notin F$, so $f(x) \geq 3^{-m} / 4$ by Lemma 2.3; if $F$ is bounded, then we can find such $m$ independent of $x$, so $0<\inf f$. Conversely if $f$ is positive and $a$ is a branch, let $x=$ $\lim _{m \rightarrow \infty} a(m)$. As $f(x)>0$ we can find $m$ such that $a(m) \notin F$; and if $0<\inf f$ we can choose $m$ independent of $a$.

Conversely suppose $f$ is a nonnegative uniformly continuous function on $[0,1]$. Let $\theta$ be a rational number strictly between $\frac{1}{2}$ and 1 . Define the map $\lambda$ from the complete binary fan $C$ to the real numbers by setting $\lambda(a)=\sum_{l=1}^{|a|} a_{l} \theta^{i-1}$. Then $\lambda$ maps $C$ onto a dense subset of the closed interval with endpoints $\pm 1 /(1-\theta)$ (compare [5; 3.3.3]). By a change of variable we may assume the given function $f$ is defined on this interval. As $f$ is uniformly continuous we can find a function $\omega$ from the positive rationals to the positive rationals such that $|f(x)-f(y)|<\omega(\delta)$ whenever $|x-y|<\delta$, and $\omega(\delta)$ goes to 0 with $\delta$. Let $B=\left\{a \in C: \omega\left(\theta^{|a|} /(1-\theta)\right)\right.$ $<f(\lambda(a))\}$. Since the relation $r_{1}<r_{2}$ for real numbers is of the form $\exists n P(n)$ for a decidable predicate $P$, the set $B$ is countable. Let $B^{\prime}$ be the detachable set associated with $B$ by Lemma 1.2, and let $F=\{a \in C$ : $a(m) \notin B^{\prime}$ for all $\left.m \leq|a|\right\}$. If $f$ is everywhere positive, then $B$, and therefore $B^{\prime}$, is a bar, so $F$ is branch-bounded; and if $0<\inf f$, then $F$ is bounded. Conversely if $F$ is branch-bounded (respectively, bounded), then $f$ is positive (respectively, bounded away from 0 ) for if the node $a$ is a restriction of the node $b$, then $f(\lambda(b))>f(\lambda(a))-\omega\left(\theta^{|a|} /(1-\theta)\right)$.

COROLlary 2.5. Assuming CPF there is a uniformly continuous function $f$ on $[0,1]$ such that $f(x)>0$ for all $x$, and the infimum of $f$ is 0 .

Proof. Theorems 1.3 and 2.4.

One consequence of Brouwer's fan theorem [5; 3.4.4] is that every branch-bounded fan is bounded. Given this we can show that every uniformly continuous positive function on $[0,1]$ has a positive infimum (compare [5; 3.4.5, Theorem 4] where the full force of the fan theorem is invoked, so the function need not be assumed continuous as it is automatically uniformly continuous).

COROLlaRY 2.6. Every detachable branch-bounded fan is bounded if and only if every positive uniformly continuous function on $[0,1]$ has a positive infimum.

Proof. Theorem 2.4. 
3. Applications. We close with three applications of this construction. Bishop calls a totally bounded set $K$ well contained in an open set $U$ if some $\delta$-neighborhood of $K$ is contained in $U$. When he raises the problem of whether every positive, uniformly continuous function on $[0,1]$ has a positive infimum he points out that this is equivalent to every compact subset of the open unit disc $U$ being well contained in $U$. But given a positive function on $[0,1]$ with infimum zero we can construct a compact subset of $U$ that has the same diameter as $U$ :

THEOREM 3.1. Assuming CPF there is a compact subset of the open unit disc of diameter 2.

Proof. Let $f$ be a positive function on $[0,1]$ with infimum 0 . The points whose polar coordinates $(r, \theta)$ satisfy $|r| \leq 1-f(\theta)$ and $0 \leq \theta \leq 1$ form a compact set of diameter 2 .

There are many constructively distinct notions of connectivity for compact metric spaces. A compact space $X$ is stepwise connected if, given any $a$ and $b$ in $X$, and $\delta>0$, there exists a sequence $a=x_{0}, x_{1}, \ldots, x_{n}=b$ such that $d\left(x_{t-1}, x_{i}\right)<\delta$ for $i=1, \ldots, n$. Alternatively we could define $X$ to be connected if whenever $X$ is the union of two nonempty open subsets $A$ and $B$, then $A$ and $B$ have a point in common. Any stepwise connected compact subset of the line is a compact interval and so [10; Theorem 2] is connected in the latter sense. This is not the case in the plane as the following example shows:

THEOREM 3.2. Assuming CPF there is a stepwise connected compact subset $X$ of the plane that is the disjoint union of two nonempty open compact subsets. In fact $X$ is the disjoint union of two Jordan arcs.

Proof. Let $f$ be a positive uniformly continuous function on $[0,1]$ with infimum 0 , and let $X=\{(x, f(x)): x \in[0,1]\} \cup[0,1] \times\{0\}$. To see that $X$ is a closed subset of the plane, suppose $(x, y)$ is in the closure of $X$. Either $y>0$, in which case $y=f(x)$, or $y<f(x)$, in which case $y=0$. The rest is easy.

Finally we give an example of a pointwise continuous function that is not uniformly continuous, but is the inverse of a uniformly continuous function:

THEOREM 3.3. Assuming CPF there is a one-to-one uniformly continuous map of the circle into the plane whose inverse is pointwise continuous but not uniformly continuous. 
Proof. Let $f$ be a positive function on $[0,1]$ with infimum 0 . Paste together the four Jordan arcs: $A_{1}=[0,1] \times\{0\}, A_{2}=\{1\} \times[0, f(1)]$, $A_{3}=\{(x, f(x)): 0 \leq x \leq 1\}$ and $A_{4}=\{0\} \times[0, f(0)]$.

\section{REFERENCES}

[1] O. Aberth, Computable Analysis, McGraw-Hill, N. Y. 1980.

[2] M. Beeson, Foundations of constructive mathematics: metamathematical studies, to appear.

[3] E. Bishop, Foundations of Constructive Analysis, McGraw-Hill, New York, 1967.

[4] A. Grzegorczyk, Computable functionals, Fund. Math., 42 (1945), 168-202.

[5] A. Heyting, Intuitionism, an Introduction (third edition), North-Holland Publishing Co., Amsterdam, 1971.

[6] S. C. Kleene, Introduction to Metamathematics, Van Nostrand, 1952.

[7] S. C. Kleene and R. E. Vesley, The Foundations of Intuitionistic Mathematics, North-Holland Publishing Co., Amsterdam, 1965.

[8] G. Kreisel, Mathematical reviews 1958, p. 238, review of H. Mechkowski, Zur rekursiven Funcktionentheorie.

[9] D. Lacombe, Remarques sur les operateurs recursifs et sur les fonctions recursives d'une variable reelle, C. R. Acad. Sci. Paris, 241 (1955), 1250-1252.

[10] M. Mandelkern, Connectivity of an interval, Proc. Amer. Math. Soc., 54 (1976), 170-172.

[11] F. Richman, Church's thesis without tears, to appear in J. Symbolic Logic.

[12] E. Specker, Nicht konstruktiv beweisbare Sätze der Analysis, J. Symbolic Logic, 14 (1949), 145-158.

[13] __ Der Satz vom Maximum in der Rekursive Analysis, Constructivity in Mathematics, Proc. Colloq. Amsterdam 1957, 254-265, North-Holland, 1959.

[14] I. D. Zaslavsky, Some properties of constructive real numbers and constructive functions, Amer. Math. Soc. Trans., 57 (1966), 1-84.

Received April 26, 1982.

New Mexico State University

Las CRUCES, NM 88003 


\title{
PACIFIC JOURNAL OF MATHEMATICS EDITORS
}

\author{
Donald BABBITT (Managing Editor) \\ University of California \\ Los Angeles, CA 90024 \\ Hugo RossI \\ University of Utah \\ Salt Lake City, UT 84112 \\ C. C. MOore and Arthur Ogus \\ University of California \\ Berkeley, CA 94720
}

J. DUGUNDJI
Department of Mathematics

University of Southern California

Los Angeles, CA 90089-1113

R. FinN and H. SAMELSON

Stanford University

Stanford, CA 94305

\section{ASSOCIATE EDITORS}
R. ARENS
E. F. BECKENBACH
B. H. NeumanN
F. WOLF
K. YosHIDA (1906-1982)

\section{SUPPORTING INSTITUTIONS}

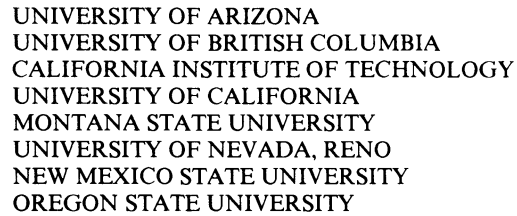

UNIVERSITY OF ARIZONA

UNIVERSITY OF BRITISH COLUMBIA

CALIFORNIA INSTITUTE OF TECHNOLOGY

UNIVERSITY OF CALIFORNIA

MONTANA STATE UNIVERSITY

UNIVERSITY OF NEVADA, RENO

NEW MEXICO STATE UNIVERSITY

OREGON STATE UNIVERSITY

\author{
UNIVERSITY OF OREGON \\ UNIVERSITY OF SOUTHERN CALIFORNIA \\ STANFORD UNIVERSITY \\ UNIVERSITY OF HAWAII \\ UNIVERSITY OF TOKYO \\ UNIVERSITY OF UTAH \\ WASHINGTON STATE UNIVERSITY \\ UNIVERSITY OF WASHINGTON
}

The Supporting Institutions listed above contribute to the cost of publication of this Journal, but they are not owners or publishers and have no responsibility for its content or policies.

Mathematical papers intended for publication in the Pacific Journal of Mathematics should be in typed form or offset-reproduced (not dittoed), double spaced with large margins. Please do not use built up fractions in the text of the manuscript. However, you may use them in the displayed equations. Underline Greek letters in red, German in green, and script in blue. The first paragraph must be capable of being used separately as a synopsis of the entire paper. In particular it should contain no bibliographic references. Please propose a heading for the odd numbered pages of less than 35 characters. Manuscripts, in triplicate, may be sent to any one of the editors. Please classify according to the scheme of Math. Reviews, Index to Vol. 39. Supply name and address of author to whom proofs should be sent. All other communications should be addressed to the managing editor, or Elaine Barth, University of California, Los Angeles, California 90024.

There are page-charges associated with articles appearing in the Pacific Journal of Mathematics. These charges are expected to be paid by the author's University, Government Agency or Company. If the author or authors do not have access to such Institutional support these charges are waived. Single authors will receive 50 free reprints; joint authors will receive a total of 100 free reprints. Additional copies may be obtained at cost in multiples of 50 .

The Pacific Journal of Mathematics is issued monthly as of January 1966. Regular subscription rate: $\$ 132.00$ a year (6 Vol., 12 issues). Special rate: $\$ 66.00$ a year to individual members of supporting institutions.

Subscriptions, orders for numbers issued in the last three calendar years, and changes of address should be sent to Pacific Journal of Mathematics, P.O. Box 969, Carmel Valley, CA 93924, U.S.A. Old back numbers obtainable from Kraus Periodicals Co., Route 100, Millwood, NY 10546.

The Pacific Journal of Mathematics ISSN 0030-8730 is published monthly by the Pacific Journal of Mathematics at P.O. Box 969, Carmel Valley, CA 93924. Application to mail at Second-class postage rates is pending at Carmel Valley, California, and additional mailing offices. Postmaster: Send address changes to Pacific Journal of Mathematics, P. O. Box 969, Carmel Valley, CA 93924.

PUBLISHED BY PACIFIC JOURNAL OF MATHEMATICS, A NON-PROFIT CORPORATION 


\section{Pacific Journal of Mathematics}

\section{Vol. 111, No. $2 \quad$ December, 1984}

Berndt Brenken, Representations and automorphisms of the irrational

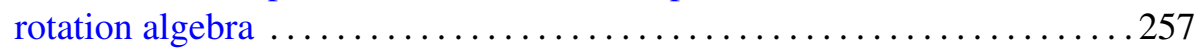

Harold George Diamond, A number theoretic series of I. Kasara . . . . . . 283

Rolf Farnsteiner, On the structure of simple-semiabelian Lie algebras . . . . 287

Guillermo Grabinsky, Poisson process over $\sigma$-finite Markov chains . ......301

Derbiau Frank Hsu and A. Donald Keedwell, Generalized complete

mappings, neofields, sequenceable groups and block designs. I . . . . . 317

William H. Julian and Fred Richman, A uniformly continuous function on

$[0,1]$ that is everywhere different from its infimum $\ldots \ldots \ldots \ldots . \ldots 33$

D. H. Lehmer and Emma Lehmer, The sextic period polynomial .........341

E. Maluta, Uniformly normal structure and related coefficients ...........357

Coy Lewis May, The species of bordered Klein surfaces with maximal

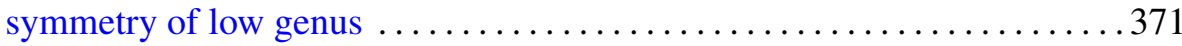

Louis Jackson Ratliff, Jr., On asymptotic prime divisors . . . . . . . . . . . 395

Norbert Riedel, Disintegration of KMS-states and reduction of standard von

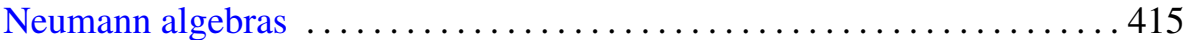

Richard Gordon Swan, $n$-generator ideals in Prüfer domains ...........433

Vilmos Totik, An interpolation theorem and its applications to positive

operators .................................. 447

Richard Vrem, Hypergroup joins and their dual objects 